\title{
Discriminación condicional de dos opciones utilizando programas definidos temporalmente*
}

\section{Two-Choice Conditional Discrimination Using Temporally-Defined Schedules Discriminação condicional de duas opções utilizando programas definidos temporalmente}

\author{
Mario Serrano** \\ Universidad Veracruzana, Veracruz, México. \\ Rodrigo Sosa ${ }^{* * *}$ \\ Universidad Iberoamericana, Ciudad de México, México. \\ Carlos Flores**** \\ Universidad de Guadalajara, Guadalajara, México.
}

Doi: http://dx.doi.org/10.12804/revistas.urosario.edu.co/apl/a.4618

\section{Resumen}

En el contexto de algunas discrepancias experimentales sobre las contingencias de disponibilidad limitada de reforzamiento y el desarrollo del control del estímulo simple y condicional, se expuso a ratas a un procedimiento de discriminación condicional de dos opciones utilizando programas definidos temporalmente. Los subciclos $\mathrm{t}^{\mathrm{D}} \mathrm{y} \mathrm{t}^{\Delta}$ tuvieron $30 \mathrm{~s}$ de duración y las señales agregadas al subciclo $\mathrm{t}^{\mathrm{D}}$ fueron auditivas o visuales. Dentro de cada subciclo $\mathrm{t}^{\mathrm{D}}$ la primera respuesta en una de dos palancas disponibles produjo el reforzamiento, si y solo si la combinación señal-palanca igualó el arreglo predefinido experimentalmente. Excepto por una rata expuesta a señales auditivas, el porcentaje de respuestas correctas incrementó progresivamente a través de las sesiones. Adicionalmente, al final del experimento la frecuencia de respuestas durante el subciclo $t^{\mathrm{D}}$ fue

* La presente investigación fue posible gracias al proyecto n. ${ }^{\circ} 180619$ del Conacyt a cargo del primer autor. La redacción final del manuscrito se realizó durante una estancia sabática del primer autor en el Centro de Estudios e Investigaciones en Comportamiento de la Universidad de Guadalajara, por invitación de Carlos Javier Flores Aguirre. Se agradece el auspicio de las condiciones necesarias para el trabajo académico al director de dicha entidad, Oscar García Leal.

** Mario Serrano, Centro de Estudios e Investigaciones en Conocimiento y Aprendizaje Humano, Universidad Veracruzana, Veracruz, México.

*** Rodrigo Sosa, Facultad de Psicología, Universidad Iberoamericana, Ciudad de México, México.

****Carlos Flores, Carlos Torres, Centro de Estudios e Investigaciones en Comportamiento, Universidad de Guadalajara, Guadalajara, México.

La correspondencia relativa a este artículo debe ser enviada a Mario Serrano, Universidad Veracruzana, Centro de Estudios e Investigaciones en Conocimiento y Aprendizaje Humano, Av. Orizaba No. 203, Fraccionamiento Veracruz, Xalapa, Veracruz, México, C. P. 91120. Correo electrónico: mserrano@uv.mx

Cómo citar este artículo: Serrano, M., Sosa, R., Flores, C. \& Torres, C. (2018). Discriminación condicional de dos opciones utilizando programas definidos temporalmente. Avances en Psicología Latinoamericana, 36(3), 527-536. DOI: http://dx.doi. org/10.12804/revistas.urosario.edu.co/apl/a.4618 
más elevada que durante el subciclo $\mathrm{t}^{\Delta}$ para todas las ratas. En línea con algunos de los estudios previos, los resultados confirman que las contingencias de disponibilidad limitada de reforzamiento no impiden el control del estímulo.

Palabras clave: discriminación condicional, disponibilidad limitada de reforzamiento, modalidad del estímulo, programas definidos temporalmente, apretar la palanca, ratas.

\section{fbstract}

Given experimental discrepancies regarding limited availability contingencies for reinforcement, and the development of control stimuli, this study exposed rats were exposed to a two-choice conditional discrimination procedure using temporally-defined schedules. Both $\mathrm{t}^{\mathrm{D}}$ and $\mathrm{t}^{\Delta}$ subcycles were $30 \mathrm{~s}$ long and signals added to the $\mathrm{t}^{\mathrm{D}}$ subcycle were auditory or visual. Within each $\mathrm{t}^{\mathrm{D}}$ subcycle the first response in either of the two available levers produced reinforcement, if and only if the signal-lever combination matched the experimental predefined arrangement. Except for one rat exposed to auditory stimuli, the percentage of correct responses progressively increased across sessions. Additionally, at the end of the experiment response frequency during the $\mathrm{t}^{\mathrm{D}}$ subcycle was higher than during the $\mathrm{t}^{\Delta}$ subcycle for all rats. In line with previous studies, results confirm that limited-hold reinforcement does not prevent stimulus control.

Keywords: Conditional discrimination, limited-hold reinforcement, stimulus modality, temporally-defined schedules, lever-pressing, rats.

\section{Resumo}

Se expôs a ratas a um procedimento de discriminação condicional de duas opções utilizando programas definidos temporalmente. Os subciclos $\mathrm{t}^{\mathrm{D}} \mathrm{e} \mathrm{t}^{\mathrm{\Delta}}$ tiveram 30 $\mathrm{s}$ de duração e os sinais agregadas ao subciclo $\mathrm{t}^{\mathrm{D}}$ foram auditivas ou visuais. Ao interior de cada subciclo $t^{\mathrm{D}} \mathrm{a}$ primeira resposta em uma de duas alavancas disponíveis produziu o reforçamento, se e só se a combinação sinal-alavanca igualou o arranjo predefinido experimentalmente. Com a exceção de uma rata exposta a sinais auditivas, a porcentagem de respostas corretas incrementou progressivamente através das sessões. Adicionalmente, no final do experimento a frequência de respostas durante o subciclo $t^{D}$ foi mais elevada que durante o subciclo $t^{\Delta}$ para todas as ratas. Em linha com estudos prévios, os resultados confirmam que a disponibilidade limitada de reforçamento não impede o controle do estímulo.

Palavras-chave: discriminação condicional, disponibilidade limitada de reforços, modalidade do estímulo, programas definidos temporalmente, apertar a alavanca, ratas.

Los programas definidos temporalmente consisten en la presentación repetida de ciclos temporales $(\mathrm{T})$ divididos en dos subciclos. El primero de ellos es el subciclo $t^{\mathrm{D}}$, periodo durante el cual la primera respuesta emitida sobre un operando produce la entrega del reforzador. De no emitirse respuesta alguna durante dicho subciclo, el reforzador programado para ese ciclo $\mathrm{T}$ particular no se presenta. El subciclo restante se denomina $\mathrm{t}^{\Delta}$, el cual se caracteriza porque la probabilidad de reforzamiento es usualmente menor que en el subciclo $t^{\mathrm{D}}$. En la mayoría de los estudios disponibles, sin embargo, las respuestas emitidas a lo largo del subciclo $\mathrm{t}^{\Delta}$ no han tenido consecuencias programadas (véase Carpio, 1990; Ribes \& Carpio, 1991; Schoenfeld \& Cole, 1972).

Aunque los programas definidos temporalmente se idearon para reproducir las ejecuciones observadas bajo programas de reforzamiento de razón e intervalo especificando solo criterios temporales (Schoenfeld, Cumming \& Hersts, 1956), más recientemente se han utilizado para evaluar el desarrollo de las funciones discriminativa y delta de los estímulos. A diferencia de los programas de reforzamiento múltiple, en los que las señales correlacionadas con el reforzamiento y la extinción de las respuestas permanecen vigentes durante toda 
la duración de los componentes (Ferster \& Skinner, 1957), en los programas definidos temporalmente la señal agregada al subciclo $\mathrm{t}^{\mathrm{D}}$ se cancela por la respuesta procuradora de reforzamiento. El resto de dicho subciclo trascurre en ausencia de señal exteroceptiva alguna, mientras que la señal correlacionada con extinción se presenta a lo largo del subciclo $\mathrm{t}^{\Delta}$. Mediante esta metodología, diversos estudios reportaron que las señales correlacionadas positiva y negativamente con el reforzamiento de las respuestas no necesariamente desarrollan las funciones discriminativa y delta correspondientes (e.g., Ribes \& Torres, 1996; Ribes \& Torres, 1997; Ribes, Torres, Barrera \& Mayoral, 1997; Ribes, Torres \& Piña, 1999; Ribes, Torres \& Mayoral, 2000a; Ribes, Torres \& Mayoral, 2000b; Ribes, Torres \& Mayoral, 2002).

Ribes et al. (2002), por ejemplo, expusieron a ratas a ciclos $\mathrm{T}$ en los que, respectivamente, los subciclos $\mathrm{t}^{\mathrm{D}}$ y $\mathrm{t}^{\Delta}$ se correlacionaron con diferentes señales exteroceptivas y probabilidades de reforzamiento de 1.00 y 0.00. Para dos ratas las señales pertenecieron a la dimensión visual y para otras dos las señales pertenecieron a la dimensión auditiva. Observaron que, independientemente del tipo de señales utilizadas, aquellas que se correlacionaron con extinción durante el subciclo $t^{\Delta}$ controlaron una frecuencia de respuesta más alta que aquellas que se correlacionaron con el reforzamiento de las respuestas en el subciclo $t^{\mathrm{D}}$. Esta ausencia de control discriminativo se atribuyó tanto a la carencia de un patrón de respuestas en presencia de la señal agregada al subciclo $t^{\mathrm{D}}$, como a la diferencia entre los reforzadores programados y los realmente obtenidos, ambos efectos derivados de la disponibilidad limitada de reforzamiento especificada por la duración de dicho subciclo.

Si la disponibilidad limitada de reforzamiento impide el desarrollo de la discriminación simple, cabe preguntarse si se observará un efecto análogo en el caso de la discriminación condicional. La respuesta a esta pregunta no es necesariamente obvia. Por un lado, existen experimentos análogos al reportado por Ribes et al. (2002) en los que la disponibilidad limitada de reforzamiento no impidió la discriminación simple. En tales estudios se observó una mayor frecuencia de respuestas en el subciclo $\mathrm{t}^{\mathrm{D}}$ que en el subciclo $\mathrm{t}^{\Delta}$ cuando las señales agregadas pertenecieron a diferentes dimensiones físicas o bien a la dimensión auditiva (Serrano, Moreno, Camacho, Aguilar \& Carpio, 2006), al igual que cuando el final del subciclo $\mathrm{t}^{\Delta}$ y el inicio del siguiente subciclo $\mathrm{t}^{\mathrm{D}}$ se separaron temporal (Mateos \& Flores, 2009) o probabilísticamente (Serrano, Sosa \& González, 2016). En el primero de estos dos estudios las señales agregadas a los subciclos pertenecieron a la dimensión visual, mientras en el segundo pertenecieron a la auditiva.

Por otro lado, y en contraste con los estudios inmediatamente anteriores, experimentos recientes en el área del ajuste comportamental (véase Serrano, 2009) sugieren que la disponibilidad limitada de reforzamiento efectivamente perjudica la precisión del responder bajo situaciones de discriminación condicional de dos opciones, es decir, aquellas en las que el reforzamiento, por responder en uno de dos operandos, está determinado por la señal exteroceptiva presentada (e.g., Trapold, 1970). Serrano (2016), por ejemplo, entre fases expuso a ratas a la entrega de agua no contingente, contingente y según una situación de discriminación condicional de dos opciones, utilizando señales pertenecientes a la dimensión auditiva y programas definidos temporalmente. Aunque bajo ninguna de las condiciones de entrega de agua se presentó señal alguna durante el subciclo $t^{\Delta}$, la duración de este y la del subciclo $\mathrm{t}^{\mathrm{D}}$ fueron equivalentes a las utilizadas por Ribes et al. (2002), es decir, se implementó una disponibilidad limitada de reforzamiento de 30 s. En la condición en la que el agua se entregó según la situación de discriminación condicional de dos opciones, se observó que la precisión del responder fue menor al nivel del azar para las tres ratas del experimento. En otro estudio, Serrano y García (2017) reportaron resultados similares 
utilizando señales pertenecientes a la dimensión visual, y programas definidos temporalmente de 60,30 y $15 \mathrm{~s}$, implementando disponibilidades limitadas de reforzamiento de 30,15 y $7.5 \mathrm{~s}$, respectivamente.

La discrepancia entre los resultados observados por Ribes y sus colaboradores (1996, 1997, 1999 , 2000a, 2000b, 2002) y los reportados por otros autores permite suponer que la baja precisión del responder en la situación de discriminación condicional de dos opciones, reportada por Serrano (2016) y por Serrano y García (2017), se haya debido no a la disponibilidad limitada de reforzamiento implementada en cada caso, sino a que las ratas enfrentaron dicha condición de entrega de agua después de dos condiciones en las que el líquido se entregó según arreglos contingenciales distintos. De ser este el caso, ratas sin historia experimental deberían mostrar ejecuciones por encima del nivel del azar, incluso independientemente de la dimensión física a la que pertenecen las señales agregadas al subciclo $\mathrm{t}^{\mathrm{D}}$. Con esto en mente, el presente estudio exploró la precisión del responder bajo una situación de discriminación condicional de dos opciones, utilizando un programa definido temporalmente y señales condicionales pertenecientes a las dimensiones auditiva o visual.

\section{Método}

\section{Sujetos}

Se utilizaron ocho ratas macho, cepa Wistar (Rattus norvegicus), experimentalmente ingenuas y de aproximadamente tres meses de edad al inicio del experimento. Las ratas se mantuvieron en un régimen de privación de agua de 22.5 horas diarias y durante todo el experimento tuvieron acceso libre al alimento. Después de cada sesión experimental las ratas tuvieron acceso libre a bebederos con agua durante 30 minutos en sus jaulas hogar, las cuales se ubicaron en una colonia con temperatura controlada y un ciclo luz-oscuridad 12:12.

\section{Aparatos}

Se utilizaron cuatro cámaras de condicionamiento operante de la marca Med Associaties Inc. ( ${ }^{(E N V-008) . ~ C a d a ~ c a ́ m a r a ~ m e d i ́ a ~} 30.5 \mathrm{~cm}$ de largo por $24.1 \mathrm{~cm}$ de ancho y $21.0 \mathrm{~cm}$ de alto. Las paredes anteriores y posteriores de las cámaras eran de aluminio y las paredes laterales eran de acrílico transparente. En el centro de la pared anterior de cada cámara, a $2 \mathrm{~cm}$ del piso de rejilla, se colocó una abertura para un dispensador de agua (ENV202M-S). Este proporcionó 0.01 cc de agua en cada activación. A $2.5 \mathrm{~cm}$ del piso de rejilla y a $2.5 \mathrm{~cm}$ de las paredes laterales izquierda y derecha, en cada caja se colocaron dos palancas (ENV-112CM) que requirieron aproximadamente una fuerza de $0.25 \mathrm{~N}$ para ser operadas. Dos centímetros arriba de cada palanca se colocó un módulo de estímulo triple (ENV-222M) provisto con leds de color rojo, amarillo y verde. A $18 \mathrm{~cm}$ del piso de rejilla se colocaron dos sonalerts que emitieron, respectivamente, un tono de $2900 \mathrm{~Hz}$ (ENV-223AM) y otro de 4500 $\mathrm{Hz}$ (ENV-233HAM) en cada activación; el primero se colocó en la esquina superior izquierda de la pared anterior de las cámaras y el segundo en la esquina superior derecha. Las cámaras se colocaron dentro de cubículos de aislamiento acústico (ENV022MD-27), provistos cada uno con un ventilador que facilitó la circulación del aire y proporcionó ruido enmascarador constante. Para la programación y registro de los eventos experimentales, se utilizó una computadora de escritorio estándar (HP Compac Pro 6305), equipada con una interfaz (SG6510DA) y software (SOF-735) especializados.

\section{Procedimiento}

Inicialmente, para todas las ratas se moldeó la respuesta de apretar la palanca izquierda - PImediante el método de aproximaciones sucesivas. Concluido el moldeamiento, las ratas fueron expuestas a dos sesiones de reforzamiento continuo, cada una de las cuales concluyó con 60 activaciones 
del dispensador de agua o después de una hora, lo que ocurriera primero. En la primera sesión únicamente se insertó la PI, mientras en la segunda solo se insertó la palanca derecha - $\mathrm{PD}$ -

En el experimento propiamente dicho, para todas las ratas se utilizó un programa definido temporalmente de $60 \mathrm{~s}$. Los primeros $30 \mathrm{~s}$ correspondieron al subciclo $\mathrm{t}^{\mathrm{D}}$ y los $30 \mathrm{~s}$ restantes al subciclo $t^{\Delta}$. La probabilidad de reforzamiento en el subciclo $t^{\mathrm{D}}$ fue de 1.00 , mientras en el subciclo $\mathrm{t}^{\Delta}$ fue de 0.00. Entre ciclos T, para las ratas R1, R2, R3 y R4 se agregó al subciclo $\mathrm{t}^{\mathrm{D}}$ un tono de $2.900 \mathrm{~Hz}$ (E1) o bien un tono de $4.500 \mathrm{~Hz}$ (E2), los cuales fueron emitidos por los sonalerts. Para las ratas R5, R6, R7 y R8 se agregaron al subciclo $\mathrm{t}^{\mathrm{D}}$ los leds rojos (E3) o bien los leds verdes (E4) ubicados encima de ambas palancas. La primera respuesta en el subciclo $t^{\mathrm{D}}$ produjo la activación del dispensador de agua durante $3 \mathrm{~s}$ y la cancelación de la señal en vigor, únicamente cuando las respuestas correspondieron con las combinaciones predefinidas experimentalmente: E1-PD y E2-PI para las ratas R1 a R4 y E3-PD y E4-PI para las ratas R5 a R8. En ambos casos, en las otras dos combinaciones la primera respuesta en el subciclo $\mathrm{t}^{\mathrm{D}}$ produjo únicamente la cancelación de las señales exteroceptivas. Cuando no se emitió respuesta alguna en el subciclo $t^{\mathrm{D}}$, las señales permanecieron encendidas hasta el final del subciclo y la entrega de agua programada para ese ciclo T particular no se presentó. Durante el subciclo $t^{\Delta}$ las respuestas emitidas no tuvieron consecuencias programadas. Cada una de las 30 sesiones del experimento estuvo conformada por 60 ciclos $\mathrm{T}$.

\section{Resultados}

La figura 1 muestra tanto el porcentaje de respuestas correctas a lo largo del experimento para las ratas expuestas a las señales auditivas (columna izquierda) y visuales (columna derecha), como la frecuencia de respuesta durante los subciclos $\mathrm{t}^{\mathrm{D}} \mathrm{y} \mathrm{t}^{\Delta}$, promedio de las últimas cinco sesiones. Se observa que con excepción de la rata $\mathrm{R} 4$, el porcentaje de aciertos incrementó progresivamente para la mayoría de las ratas. En lo referente a las frecuencias de respuesta, los numerales en la esquina inferior izquierda de cada panel indican que hacia el final del experimento se emitieron más respuestas en el subciclo $\mathrm{t}^{\mathrm{D}}$ que en el subciclo $\mathrm{t}^{\Delta}$ para todas las ratas. Los numerales también indican que, globalmente, las señales visuales controlaron una mayor frecuencia de respuestas que las señales auditivas.

\section{Discusión}

Diversos estudios que evaluaron el desarrollo de la discriminación simple utilizando programas definidos temporalmente reportaron que los efectos colaterales de la disponibilidad limitada de reforzamiento que caracteriza a dichos programas, es decir la pérdida potencial de reforzadores y la ausencia de un patrón de respuestas en presencia de la señal agregada al subciclo $t^{\mathrm{D}}$, impiden la diferenciación del responder en torno de los estímulos correlacionados positiva y negativamente con el reforzamiento de las respuestas (e.g., Ribes et al., 2002). Estudios conducidos en otros laboratorios, sin embargo, observaron que la disponibilidad limitada de reforzamiento no necesariamente impide el desarrollo del control del estímulo, al menos no cuando las señales correlacionadas con cada subciclo pertenecen a la dimensión auditiva o bien a las dimensiones auditiva y visual (Serrano et al., 2006), así como cuando se interpone un intervalo entre ciclos T (Mateos \& Flores, 2009), o bien cuando los subciclos $\mathrm{t}^{\mathrm{D}} \mathrm{y} \mathrm{t}^{\Delta}$ se presentan aleatoriamente al interior de la sesión experimental (Serrano et al., 2016). Los resultados del presente experimento concuerdan con los reportados en estos tres últimos estudios, extendiendo la posibilidad del desarrollo de control del estímulo bajo condiciones de disponibilidad limitada de reforzamiento al caso de las situaciones de discriminación condicional de dos opciones. Específicamente, en el experimento que aquí se reporta se encontró que la precisión 


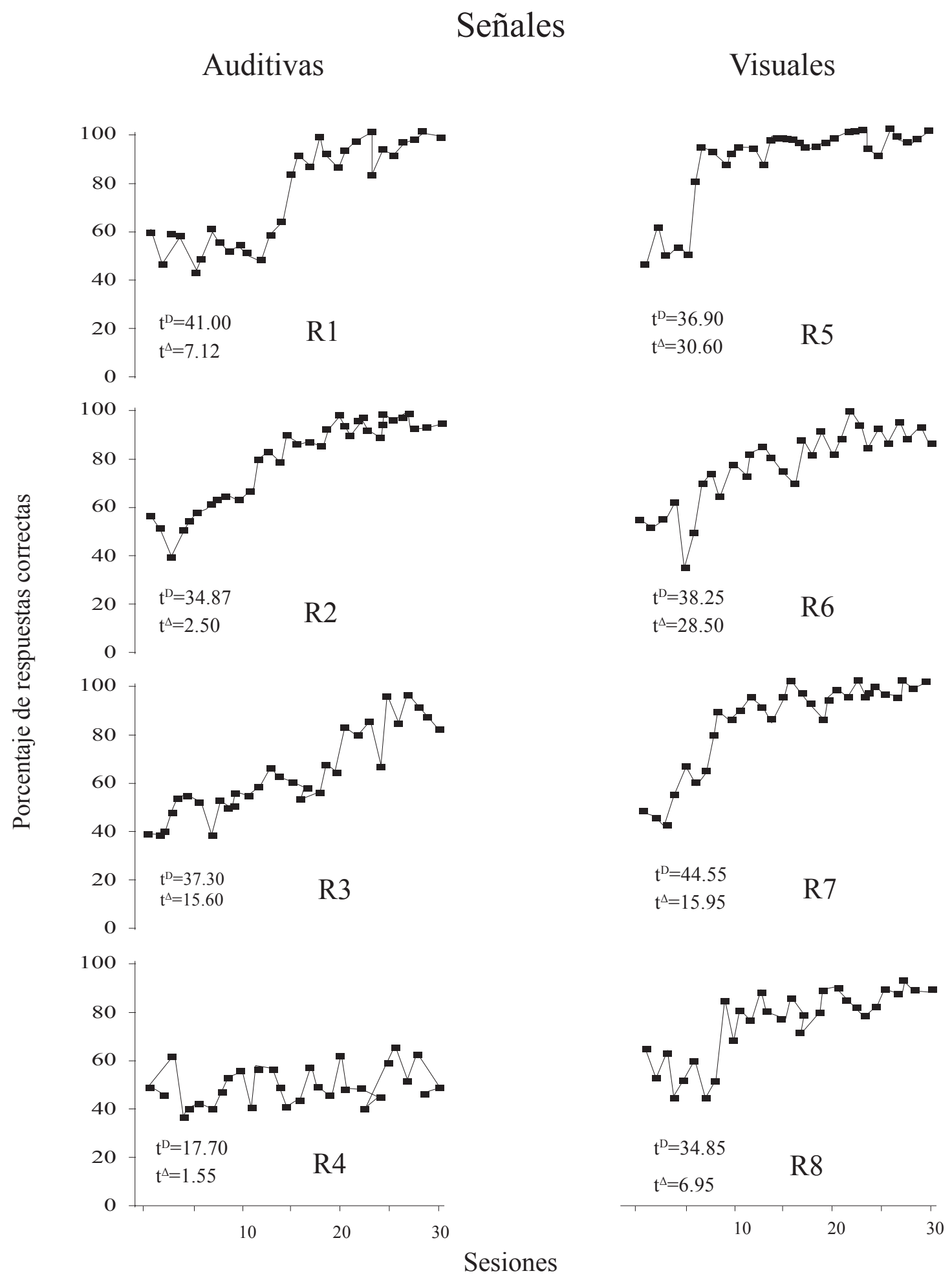

Figura 1. Porcentajes individuales de respuestas correctas en cada sesión del experimento para las ratas expuestas a señales pertenecientes a la dimensión auditiva (izquierda) y la dimensión visual (derecha). Las leyendas numéricas a la izquierda de los identificadores de cada rata se refieren al promedio de respuestas en los subciclos $\mathrm{t}^{\mathrm{D}} \mathrm{y} \mathrm{t}^{\mathrm{A}}$ en las últimas cinco sesiones del estudio 
del responder incrementó progresivamente hasta superar el $80 \%$ de respuestas correctas para la mayoría de las ratas y, adicionalmente, que hacia el final del estudio para todas ellas el responder fue mayor durante el subciclo $\mathrm{t}^{\mathrm{D}}$ que durante el subciclo $t^{\Delta}$. Estos resultados fueron relativamente independientes de la dimensión física a la que pertenecieron las señales agregadas al subciclo $t^{\mathrm{D}}$ $\mathrm{y}$, en esa medida, indican que la falta de precisión del responder bajo situaciones de discriminación condicional de dos opciones, reportada por Serrano (2016) y Serrano y García (2017), es atribuible no a los diferentes valores de disponibilidad limitada de reforzamiento implementados en cada caso, sino a los arreglos contingenciales enfrentados previamente por las ratas en ambos estudios.

Con excepción de una de las ratas expuestas a señales pertenecientes a la dimensión auditiva, la rata $\mathrm{R} 4$, los porcentajes de respuestas correctas - y por tanto las entregas de agua obtenidasincrementaron progresivamente a lo largo de las sesiones experimentales del presente estudio. Este resultado contrasta ampliamente con la mayoría de los experimentos sobre control discriminativo simple conducidos por Ribes y sus colaboradores (1996, 1997, 1999, 2000a, 2000b, 2002), en los que se observó que el porcentaje de entregas de agua obtenidas fue constantemente bajo. Es posible que tal diferencia se haya debido a las sesiones iniciales de reforzamiento continuo implementadas antes de iniciar los experimentos propiamente dichos. En el experimento reportado por Ribes et al. (2002), por ejemplo, se condujo solamente una sesión de reforzamiento continuo después del moldeamiento de la respuesta de apretar la palanca, mientras que en el estudio aquí reportado se condujeron dos sesiones de reforzamiento continuo después del moldeamiento, una sesión por cada palanca, con la finalidad de favorecer la alternación entre ellas. A pesar de que en los experimentos conducidos por Ribes y sus colaboradores los bajos porcentajes de entregas de agua obtenidas hayan sido el resultado de un efecto de estiramiento de la razón (Keller \&
Schoenfeld, 1950), esto no necesariamente vincula a los reforzadores "perdidos" con la ausencia de control por parte de la señal agregada al subciclo $\mathrm{t}^{\mathrm{D}}$. Para la ya mencionada rata $\mathrm{R} 4$, por ejemplo, el porcentaje de entregas de agua obtenidas se mantuvo alrededor del $50 \%$ a lo largo del experimento, implicando un tránsito del reforzamiento continuo a ciclos T de $120 \mathrm{~s}$ aproximadamente y, no obstante, para dicha rata se observó que el promedio final de respuestas de palanqueo también fue mayor en el subciclo $t^{\mathrm{D}}$ que en el subciclo $t^{\Delta}$.

El hecho de que en el presente experimento la disponibilidad limitada de reforzamiento no haya tenido un efecto negativo sobre la adquisición de la discriminación condicional para la mayoría de las ratas no solo es consistente con los estudios en los que se utilizaron programas definidos temporalmente con señales agregadas a los subciclos $\mathrm{t}^{\mathrm{D}}$ $\mathrm{y} \mathrm{t}^{\Delta}$ y se observó el establecimiento de la discriminación simple. Los resultados aquí reportados son igualmente consistentes con numerosos estudios en los que los procedimientos que auspiciaron el desarrollo de la discriminación condicional no implicaron disponibilidad limitada de reforzamiento alguna; por ejemplo, aquellos experimentos en los que se utilizó alguna de las diferentes versiones del procedimiento de igualación de la muestra (Skinner, 1950). Sobre estos procedimientos en particular, debe destacarse que si bien el experimentador usualmente no impone límite temporal alguno para que los sujetos respondan a los llamados estímulos comparativos, tanto las respuestas que igualan al estímulo de muestra según el criterio predefinido como las que no lo hacen, en la mayoría de los casos cancelan la presentación de todas las señales vigentes en ese momento. Dicho de otra manera, y en contraste con lo señalado por Ribes et al. (2002) en torno a la ausencia de control discriminativo bajo condiciones de disponibilidad limitada de reforzamiento, en los procedimientos de igualación de la muestra por lo general no tiene lugar un patrón de respuestas en presencia de los estímulos inmediatamente anteriores a la presentación del 
reforzador. De hecho, salvo aquellos estudios en los que se impuso algún requisito de respuesta explícito (e.g., Cohen, Brady \& Lowry, 1981), en la mayoría de los experimentos también la primera respuesta al estímulo de muestra produjo la presentación de los estímulos comparativos (véase Cumming \& Berryman, 1965; Carter \& Werner, 1978; Goeters, Blakely \& Polling, 1992; Mackey, 1991; Wixted, 1989).

En lo que a la dimensión física de las señales agregadas se refiere, destaca que, por un lado, si bien en el presente estudio la precisión de la ejecución incrementó de manera similar bajo ambos tipos de señales, el porcentaje de respuestas correctas se mantuvo alrededor del nivel del azar para una de las ratas expuestas a las señales auditivas, $y$, por el otro, que hacia el final del experimento estas hayan controlado un menor número de respuestas totales en comparación con las señales visuales. Aunque los dos puntos anteriores parecen cuestionar la idea relativamente generalizada de que las ratas son más sensibles a la estimulación auditiva que a la visual (e.g., Harrison, 1994), una explicación más simple sobre ambos aspectos radica en el hecho de que mientras los leds que emitieron las señales de la dimensión visual se ubicaron encima de las palancas de respuesta, los sonalerts que emitieron las señales de la dimensión auditiva se colocaron $18 \mathrm{~cm}$ por encima del piso de rejilla. Otra posibilidad igualmente ubicable como una limitación metodológica del presente estudio radica en un sesgo de posición debido a la falta de contrabalanceo de las señales exteroceptivas y las palancas. Al igual que la ausencia de señales durante los subciclos $\mathrm{t}^{\Delta}$, dicha omisión obedeció a la necesidad de implementar condiciones experimentales análogas a las de los estudios conducidos por Serrano (2016) y por Serrano y García (2017), con la finalidad de aislar el papel jugado por las condiciones de entrega de agua que precedieron a la situación de discriminación condicional de dos opciones en ambos estudios. Si bien futuras investigaciones idealmente no deberán omitir el contrabalanceo entre las señales y las palancas de respuesta, el incremento progresivo en la precisión del responder observado para la mayoría de las ratas del presente estudio sugiere que las sesiones iniciales de reforzamiento continuo en cada una de las palancas disminuyeron sensiblemente posibles sesgos posicionales.

Finalmente, en la medida en que la frecuencia terminal de respuestas fue mayor en el subciclo $t^{\mathrm{D}}$ que en el subciclo $t^{\Delta}$ para todas las ratas del presente estudio, y por el hecho de que esta discrepancia con los experimentos conducidos por Ribes y sus colaboradores podría atribuirse a la ausencia de una señal agregada al subciclo $\mathrm{t}^{\Delta}$, debe señalarse que si bien Ribes et al. (2002) reportaron que omitir la señal correlacionada con dicho subciclo produjo una disminución del responder en ese periodo - favoreciendo así un mayor número de respuestas en $\mathrm{t}^{\mathrm{D}}$ que en $\mathrm{t}$ - la omisión de la señal durante las últimas 25 sesiones del estudio no se vio correlacionada con un aumento en las entregas de agua. En ese contexto, puede aventurarse que, si bien la ausencia de una señal correlacionada con el subciclo $t^{\Delta}$ podría explicar la diferencia en las frecuencias de respuesta entre los subciclos, los incrementos progresivos en el porcentaje de respuestas correctas no parecen tener relación directa con dicha variable. De hecho, la literatura en el área de la discriminación condicional sugiere que más que por la presencia de una señal en el subciclo $t^{\Delta}$, la precisión del responder debería estar regulada por la duración de dicho subciclo, es decir, por la duración del intervalo entre ensayos (e.g., Nelson \& Waserman, 1978). Nuevos estudios sobre los efectos potenciales de la disponibilidad limitada de reforzamiento deberán enfocarse en dicha posibilidad.

\section{Referencias}

Carpio, C. (1990). Una aproximación paramétrica al análisis de la conducta. Revista Mexicana de Análisis de la Conducta, 16, 65-83. 
Carter, D. E. \& Werner, T. J. (1978). Complex learning and information processes by pigeons: a critical analysis. Journal of the Experimental Analysis of Behavior, 29, 565-601. DOI: 10.1901/ jeab.1978.29.565

Cohen, L. R., Brady, J. \& Lowry, M. (1981). The role of differential responding in matching-to-sample and delayed matching performance. En M. L. Commons \& J. A. Nevin (eds.), Quantitative analysis of behavior: discrimination proprieties of reinforcement schedules (pp. 345-364). Cambridge, Massachusetts: Ballinger Publishing Company.

Cumming, W. W. \& Berryman, R. (1965). The complex discriminated operant: studies of matching-to-sample and related problems. En D. I. Mostofsky (ed.), Stimulus generalization (pp. 28-330). Standford, CA: Standford University Press.

Ferster, C. B. \& Skinner, B. F. (1957). Schedules of reinforcement. Nueva York: Appleton Century Crofts.

Goeters, S., Blakely, E. \& Polling, A. (1992). Differential outcomes effect. The Psychological Record, 42, 389-412.

Harrison, J. M. (1994). The representative animal. The Behavior Analyst, 17, 207-219.

Keller, F. S. \& Schoenfeld, W. N. (1950). Principles of psychology. Nueva York: Appleton Century Crofts.

Mackey, H. A. (1991). Conditional stimulus control. En I. H. Iversen \& K. A. Lattal (eds.), Experimental analysis of behavior (pp. 301-350). Amsterdam: Elservier.

Mateos, R. \& Flores, C. J. (2009). Efectos del intervalo entre ciclos y control del estímulo en programas definidos temporalmente. Revista Mexicana de Análisis de la Conducta, 35, 101-116.

Nelson, K. R. \& Wasserman, E. A. (1978). Temporal factors influencing the pigeon's successive matching-to-sample performance: sample duration, intertrial interval, and retention interval. Journal of the Experimental Analysis of Behavior, 30, 153-62. DOI: 10.1901/JEAB.1978.30-153

Ribes, E. \& Carpio, C. (1991). Análisis de los parámetros de estímulo que regulan la conducta animal. En V. Colotla (ed.), La investigación del comportamiento en México (pp. 184-210). México: AIC/Conacyt/SMAC/UnAM.

Ribes, E. \& Torres, C. (1996). Efectos de la variación en la probabilidad de reforzamiento correlacionada con dos estímulos neutros en un programa definido temporalmente. Revista Mexicana de Análisis de la Conducta, 22, 41-78.

Ribes, E. \& Torres, C. (1997). Stimulus and functions: lack of discrimination or excess of generalization? Revista Mexicana de Análisis de la Conducta, 23, 249-274.

Ribes, E., Torres, C., Barrera J. A. \& Mayoral, A. (1997). Control discriminativo con estímulos compuestos en programas temporales de reforzamiento. Revista Mexicana de Análisis de la Conducta, 23, 25-51.

Ribes, E., Torres, C. \& Piña, J. (1999). Comparación de los efectos de la presencia y ausencia de estímulos diferenciales en dos programas con probabilidades variantes igualadas. Acta Comportamentalia, 7, 5-29.

Ribes, E., Torres, C. \& Mayoral, A. (2000a). Señalización no diferencial de distintas probabilidades de entrega de agua en dos subciclos de un programa definido temporalmente. Acta Comportamentalia, 8, 5-21.

Ribes, E., Torres, C. \& Mayoral, A. (2000b). Efectos de la ausencia y presencia de estímulos correlacionados con distintas probabilidades de reforzamiento y con extinción en programas definidos temporalmente. Revista Mexicana de Análisis de la Conducta, 26, 327-354.

Ribes, E., Torres, C. \& Mayoral, A. (2002). Extended exposure to a discriminated, limited-hold temporal Schedule does not produce stimulus control. Behavioural Processes, 59, 131-146. DOI: 10.1016/S0376-6357(02)00090-6 
Schoenfeld, W. N. \& Cole, B. K. (1972). Stimulus schedules: the t- $\tau$ system. Nueva York: Harper and Row.

Schoenfeld, W. N., Cumming, W. W. \& Hearst, E. (1956). On the classification of reinforcement schedules. Proceedings of the National Academy of Sciences, 42, 563-570.

Serrano, M. (2016). Ajuste comportamental bajo diferentes condiciones de entrega de agua. Acta Comportamentalia, 24, 5-13.

Serrano, M. (2009). Complejidad e inclusividad progresivas: algunas implicaciones y evidencias empíricas en el caso de las funciones contextual, suplementaria y selectora. Revista Mexicana de Análisis de la Conducta, 35(Monográfico), 161-178.

Serrano, M. \& García, G. (2017). Efectos de la longitud del ciclo sobre el ajuste comportamental. Revista Argentina de Ciencias del Comportamiento, 9, 5-18.
Serrano, M., Moreno, S., Camacho, I., Aguilar, F. \& Carpio, C. (2006). Dimensión física de las señales agregadas en programas definidos temporalmente. Revista Mexicana de Análisis de la Conducta, 32, 13-25.

Serrano, M., Sosa, R. \& González, C. (2016). Control del estímulo en programas fijos y variables definidos temporalmente. Acta Colombiana de Psicología, 19, 13-20. DOI: 10.14718/ acP.2016.19.2.210.14718/ACP.2015.18.2.2

Skinner, B. F. (1950). Are theories of learning necessary? Psychological Review, 57, 193-216.

Trapold, M. A. (1970). Are expectancies based upon different positive reinforcing events discriminably different? Learning and Motivation, 1, 129-140. DOI: 10.1016/0023-9690(70)90079-2

Wixted, J. T. (1989). Nonhuman short-term memory: a quantitative reanalysis of selected findings. Journal of the Experimental Analysis of Behavior, 52, 409-426. DOI: 10.1901. jeab.1989.52-409

\section{Recibido: febrero 17, 2016 Aprobado: abril 11, 2018}

